\title{
Geodesics on Regular Polyhedra with Endpoints at the Vertices
}

\author{
Dmitry Fuchs ${ }^{1}$
}

To Sergei Tabachnikov on the occasion of his 60th birthday

Received: 3 October 2015 / Revised: 23 October 2015 / Accepted: 3 March 2016 /

Published online: 23 March 2016

(C) Institute for Mathematical Sciences (IMS), Stony Brook University, NY 2016

\begin{abstract}
In a recent work of Davis et al. (2016), the authors consider geodesics on regular polyhedra which begin and end at vertices (and do not touch other vertices). The cases of regular tetrahedra and cubes are considered. The authors prove that (in these cases) a geodesic as above never begins at ends at the same vertex and compute the probabilities with which a geodesic emanating from a given vertex ends at every other vertex. The main observation of the present article is that there exists a close relation between the problem considered in Davis et al. (2016) and the problem of classification of closed geodesics on regular polyhedra considered in articles (Fuchs and Fuchs, Mosc Math J 7:265-279, 2007; Fuchs, Geom Dedic 170:319-333, 2014). This approach yields different proofs of result of Davis et al. (2016) and permits to obtain similar results for regular octahedra and icosahedra (in particular, such a geodesic never ends where it begins).
\end{abstract}

Keywords Regular polyhedra $\cdot$ Geodesic segments · Endpoints

\section{Introduction}

A geodesic on a surface of a polyhedron is, by definition, a locally shortest curve which may transversally intersect edges, but does not contain vertices besides, possibly, the endpoints. A geodesic is straight within the faces and at every intersection with an edge, the opposite angles formed by the geodesic and the edge (in the two faces attached to this edge) are equal. If a geodesic has two endpoints at vertices, we call it a simple geodesic segment. (A simple geodesic segment is allowed to have self-intersections.)

\footnotetext{
$\triangle$ Dmitry Fuchs

fuchs@math.ucdavis.edu

1 University of California, Davis, USA
} 


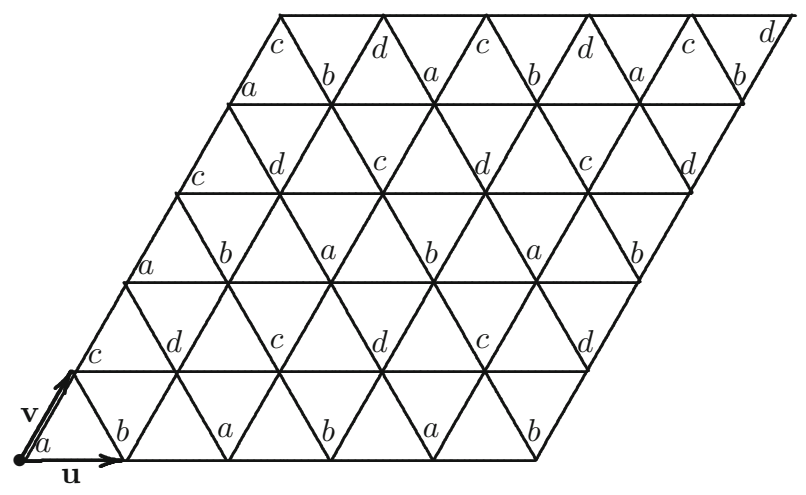

Fig. 1 The planar development of a regular tetrahedron

All the results of Davis et al. (2016) mentioned below are given with proofs, which, at least in the case of the cube, are not the same as in Davis et al. (2016).

\section{Tetrahedon}

Theorem 2.1 (Davis et al. 2016, Corollary 3.8) A simple geodesic segment starting at some vertex of a regular tetrahedron never ends at the same vertex and ends at the three other vertices with equal probabilities. ${ }^{1}$

Proof Consider the planar development of the regular tetrahedron with vertices $a, b, c, d$ (see Fig. 1). A line segment in this plane starting at the leftmost point marked $a$ and ending at one of the vertices within the angle shown in Fig. 1 has the form $p \mathbf{u}+q \mathbf{v}$ where $p$ and $q$ are non-negative integers. Namely, it ends at a vertex marked $a$, if $p$ and $q$ are both even, at a vertex marked $b$, if $p$ is odd and $q$ is even, at a vertex marked $c$, if $p$ is even and $q$ is odd, and at a vertex marked $d$, if $p$ and $q$ are both odd. It corresponds to a simple geodesic segment if and only if $p$ and $q$ are relatively prime; in particular, if it ends at $a$, then it does not correspond to any simple geodesic segment. Theorem follows.

\section{Octahedron}

Theorem 3.1 A simple geodesic segment starting at some vertex of a regular octahedron never ends at the same vertex. It ends at the opposite vertex with the probability $\frac{1}{4}$ and at the each of the other vertices with the probability $\frac{3}{16}$.

Proof Figure 2 shows a regular octrahedron (left) and its planar development. The latter is multivalued in the sense that every vertex $a, b, c$ is, actually, two vertices ( $a$

\footnotetext{
${ }^{1}$ Here and below, speaking of the probability with which a geodesic segment with a given starting point ends at some vertex, I mean the asymptotic probability for the set of geodesics of bounded length.
} 


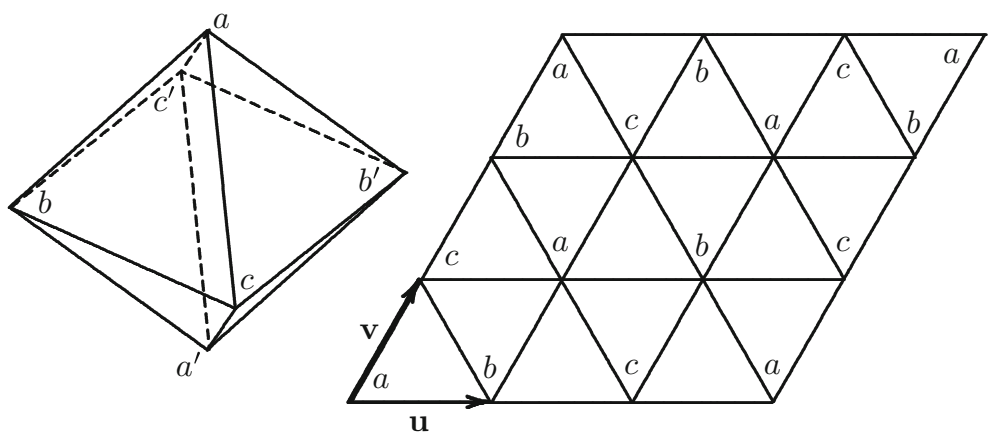

Fig. 2 A regular octahedron and its (multivalued) planar development

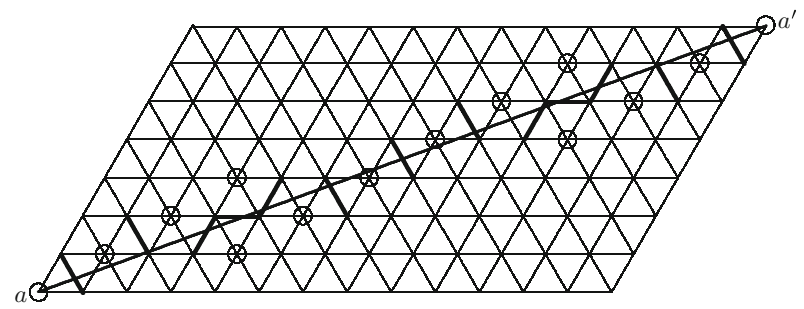

Fig. 3 The segment $p \mathbf{u}+q \mathbf{v}$ covered by the triangles of the tiling

represents $a$ and $a^{\prime}$, etc.), every edge represents four edges ( $a b$ represents $a b, a^{\prime} b, a b^{\prime}$, and $a^{\prime} b^{\prime}$, etc.), and every triangular face represents eight triangular faces. Thus every line in the plane emanating, say, from the leftmost vertex $a$ and not following the edges represents a (polygonal) line on the surface of the octahedron, but following this line we may need to replace $a$ by $a^{\prime}, b$ by $b^{\prime}$, and/or $c$ by $c^{\prime}$.

For positive relatively prime $p, q$, a vector $p \mathbf{u}+q \mathbf{v}$ represents a simple geodesic segment on the surface ending at $a$ or $a^{\prime}$, if $p-q \equiv 0 \bmod 3$, ending at $b$ or $b^{\prime}$, if $p-q \equiv 1 \bmod 3$ and ending at $c$ or $c^{\prime}$, if $p-q \equiv-1 \bmod 3$. The most importrant thing we need to prove is that it never ends at $a$.

Let $p>q>0, \operatorname{GCD}(p, q)=1, p \equiv q \bmod 3$. Figure 3 shows a parallelogram spanned by $p \mathbf{u}$ and $q \mathbf{v}$ (on the picture, $p=13, q=7$ ). We consider the diagonal segment covered by triangles of the tiling. The vertices are labeled according to the following rules. The left lower triangle is $a b c$; if two triangles share a side, then the two vertices not on this side are labeled by the same letter, one with prime, one without prime, like $a$ and $a^{\prime}, a^{\prime}$ and $a$, etc. We are interested in vertices labeled by $a$ or $a^{\prime}$. The form a sequence $a, a^{\prime}, a, a^{\prime}, \ldots$ where the neighbors are vertices of the triangles sharing a side not containing these vertices. These sides are marked (encircled) on the picture. We need to show that the number of marked sides is odd. It is 13 on the picture. Let us consider the general case.

Let $r=\frac{q}{p-q}$. The following segments are marked (coordinates are given in the basis $\{\mathbf{u}, \mathbf{v}\})$ : 


$$
\begin{aligned}
& (0,1)-(1,0) \\
& (1,2)-(2,1) \\
& ([r],[r]+1)-([r+1],[r]) \\
& ([r]+2,[r])-([r]+2,[r]+1)-([r]+3,[r]+1) \\
& \ldots([2 r]+2,[2 r])-([2 r]+2,[2 r]+1) \\
& \left.\begin{array}{c}
([2 r]+3,[2 r]+1)-([2 r]+4,[2 r]) \\
\cdots \ldots \ldots \\
([4 r]+3,[4 r]+1)-([4 r]+4,[4 r])
\end{array}\right\} \\
& ([4 r]+5,[4 r])-([4 r]+5,[4 r+1]) \ldots([5 r]+5,[5 r])-([5 r]+5,[5 r]+1) \\
& ([(p-q-4) r]+p-q-3,[(p-q-4) r]+1)-] \\
& ([(p-q-4) r+p-q-2,[(p-q-4) r]) \\
& ([(p-q-2) r]+p-q-3,[(p-q-2) r]+1)- \\
& ([(p-q-2) r]+p-q-2,[(p-q-2) r])] \\
& ([(p-q-2) r]+p-q+3,[(p+q-2)])-\cdots \\
& -([(p-q-1) r]+p-q-1,[(p+q-1) r]+1) \\
& ([(p-q-1) r]+p-q,[(p-q-1) r]+1)-] \\
& ([(p-q-1) r]+p-q,[(p-q-1) r]) \\
& ([(p-q) r]+p-q-1,[(p-q) r])-([(p-q) r]+p-q,[(p-q) r]-1)
\end{aligned}
$$

[the last segment is, actually, $(p-1, q)-(p, q-1)]$. In this listing of segments, we see the alternating of groups of parallel segments and stair-like chains. The groups of parallel segments contain, respectively, $[r]+1,[4 r]+[2 r]+1, \ldots,[(p-q-2) r]-$ $(p-q-4) r]+1, q-[(p-q-1) r]$ items; the stair-like chains contain, respectively, $2([2 r]-[r])+1,2([5 r]-[4 r])+1, \ldots, 2([(p+q-1) r]-[(p+q-2) r]+1$ items. The total is

$-[r]+[2 r]-[4 r]+[5 r]-\ldots[(p-q-2) r]+[(p-q-1) r]+q+1+\cdots+1$

where the number of 1's is $\frac{p-q}{3}$. A simple computation shows that this sum is $2\left(\left[\frac{p}{3}\right]+\left[\frac{q}{3}\right]\right)+1$, which is certainly odd.

We arrive at the following result. No simple geodesic segment emanating from $a$ ends at $a$. If this segment is determined by relatively prime $p, q$, then it ends at $a^{\prime}$ 

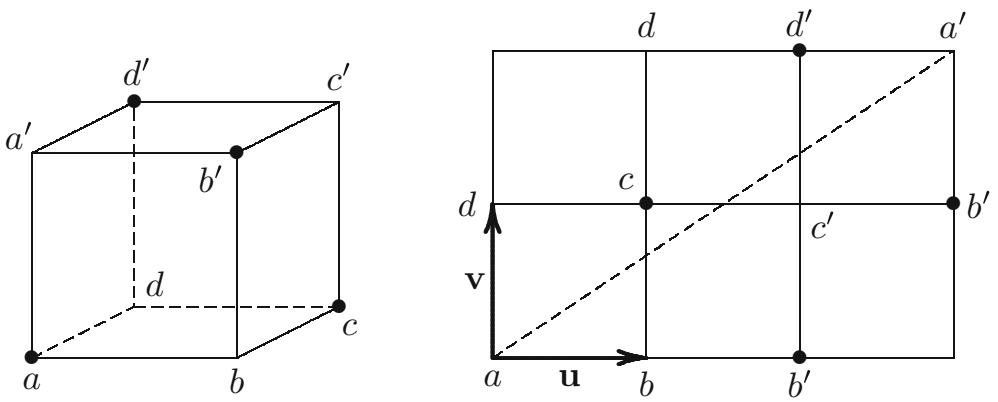

Fig. 4 The cube and a planar development of the segment $p \cdot \mathbf{u}+q \cdot \mathbf{v}$ with $p=3, q=2$

if and only if $p \equiv q \bmod 3$; asymptotically, the probability of this event is $\frac{1}{4}$. The remaining $\frac{3}{4}$ is equally (because of the symmetry) distributed between $b, b^{\prime}, c$, and $c^{\prime}$.

\section{Cube}

We begin this section with formulating some results from Fuchs and Fuchs (2007) and Fuchs (2014).

We label the vertices of the cube as shown in Fig. 4, left. An arbitrary curve on the surface of the cube not passing through the vertices may be presented as a curve in the plane furnished with the standard square lattice, and there arises a labeling of the vertices along this curve. Important remark: vertices of the lattice whose coordinates have the same parity get the labels from the set $\left\{a, b^{\prime}, c, d^{\prime}\right\}$, and vertices whose coordinates have different parities get the labels from the set $\left\{a^{\prime}, b, c^{\prime}, d\right\}$ (marked in Fig. 4).

Straight segments in the planar development correspond to geodesics on the surface of the cube. A segment $[(a, b),(a+p, b+q)]$ with integer $a, b$ and relatively prime $p, q$ corresponds to a simple geodesic segment on the cube joining two vertices of the cube, and all such geodesic segments can be obtained in this way.

If we shift the segment to a parallel segment starting at the point $(\varepsilon, 0)$ with a small $\varepsilon>0$, then we get a geodesic on the cube, which may be (and, actually, always is) not closed: the segment in Fig. 4, right, shifted a little bit to the right, corresponds to a geodesic starting near $a$ and ending near $a^{\prime}$. To get a closed geodesic, we need to repeat the segment on the plane a certain (minimal) number $n_{C}(p, q)$ of times.

Theorem 4.1 (Fuchs and Fuchs 2007, Theorem 4.4)

(1) For all $p, q, n_{C}(p, q)=2,3$, or 4 ;

(2) if $p$ and $q$ are both odd, then $n_{C}(p, q)=3$;

(3) if one of $p, q$ is even, then $n_{C}(p, q)=2$ or 4 .

To formulate a necessary result from Fuchs (2014), we need some notation. Let $\mathcal{S}$ be the set of pairs $(p, q)$ of integers such that $p$ is even, $q$ is odd, and $p, q$ do not 
Fig. 5 The function $V(p, q)$

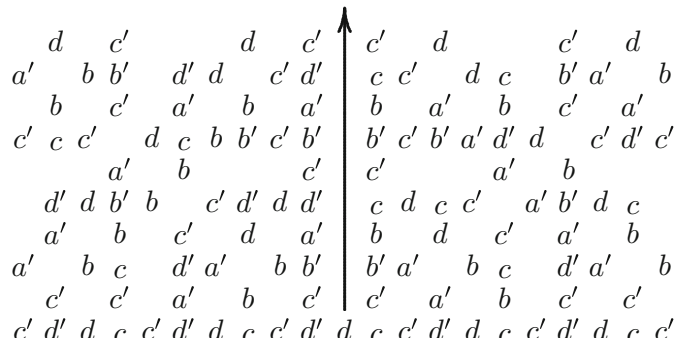

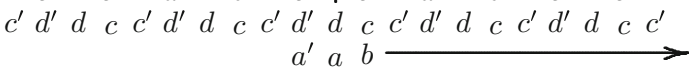

$$
\begin{aligned}
& \begin{array}{lllllllllllllllllllll}
c^{\prime} & b^{\prime} & b & c & c^{\prime} & b^{\prime} & b & c & c^{\prime} / a^{\prime} & b^{\prime} & c^{\prime} & d^{\prime} & a^{\prime} & b^{\prime} & c^{\prime} & d^{\prime} & a^{\prime} & b^{\prime} & c^{\prime}
\end{array}
\end{aligned}
$$

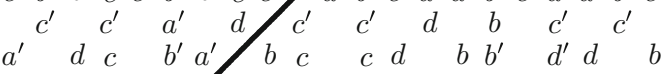

$$
\begin{aligned}
& \begin{array}{llllllllll}
a^{\prime} & d & c^{\prime} & a^{\prime} & d & b & a^{\prime} & c^{\prime} & d & b
\end{array}
\end{aligned}
$$

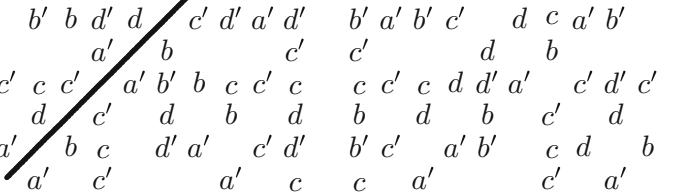

have common divisors $>1$ [thus, for example, $(0,1),(2,1) \in \mathcal{S}$, but $(0,3) \notin \mathcal{S}$ ]. The group $\Gamma_{2}$ of integer $2 \times 2$ matrices congruent to the identity modulo 2 acts transitively in $\mathcal{S}$; it is known that $\Gamma_{2}$ is a free group with generators $A=\left[\begin{array}{ll}1 & 2 \\ 0 & 1\end{array}\right], B=\left[\begin{array}{ll}1 & 0 \\ 2 & 1\end{array}\right]$. Let $H \subset \Gamma_{2}$ be the subgroup generated by $A^{2}, B^{2}, A B A$ and $B A B$. It is proved in Fuchs (2014) that $H$ has index 3 in $\Gamma_{2}$ and not normal.

Theorem 4.2 [Fuchs 2014, Theorem 2.2, Part (3)] The group $H$ has two orbits in $\mathcal{S}$, and these orbits are $\left\{n_{C}(p, q)=2\right\}$ and $\left\{n_{C}(p, q)=4\right\}$. The asymptotic size of the second of these orbits is twice the asymptotic size of the first one.

We will need here some additional facts. For every pair $(p, q)$ of relatively prime integers the segment $p \mathbf{u}+q \mathbf{v}$ determines a simple geodesic segment on the surface of the cube starting at $a$. Denote by $V(p, q)$ the endpoint of this geodesic. For example, Fig. 4 shows that $V(3,2)=a^{\prime}$. A small table of values of the function $V$ is shown in Fig. 5.

The function $V$ has a lot of symmetries, both visible and hidden. First of all, if, for a given $(p, q)$, the pair $\left(p^{\prime}, q^{\prime}\right)$ is one of $(-p, q),(p,-q),(q, p)$ or $(-q,-p)$, then $V\left(p^{\prime}, q^{\prime}\right)$ is easily related to $V(p, q)$. For example, if $q>0$, then $V(-p, q)$ is obtained from $V(p, q)$ by the transformation $a^{\prime} \leftrightarrow b, c \leftrightarrow d^{\prime}$ (indeed, all we need for this transition, is the replacement of the square $\overline{\left|\begin{array}{ll}d & c \\ a & b\end{array}\right|}$ by the square $\overline{\begin{array}{ll}d & d^{\prime} \\ a & a^{\prime}\end{array} \mid}$ ) etc. It is important that all these transformations preserve $a$ (and $c^{\prime}$ ).

To describe some other symmetries, consider the following two matrices from $H$ : $C=B^{2}=\left[\begin{array}{ll}1 & 0 \\ 4 & 1\end{array}\right], D=-A B^{-1} A=\left[\begin{array}{ll}3 & 4 \\ 2 & 3\end{array}\right]$. 

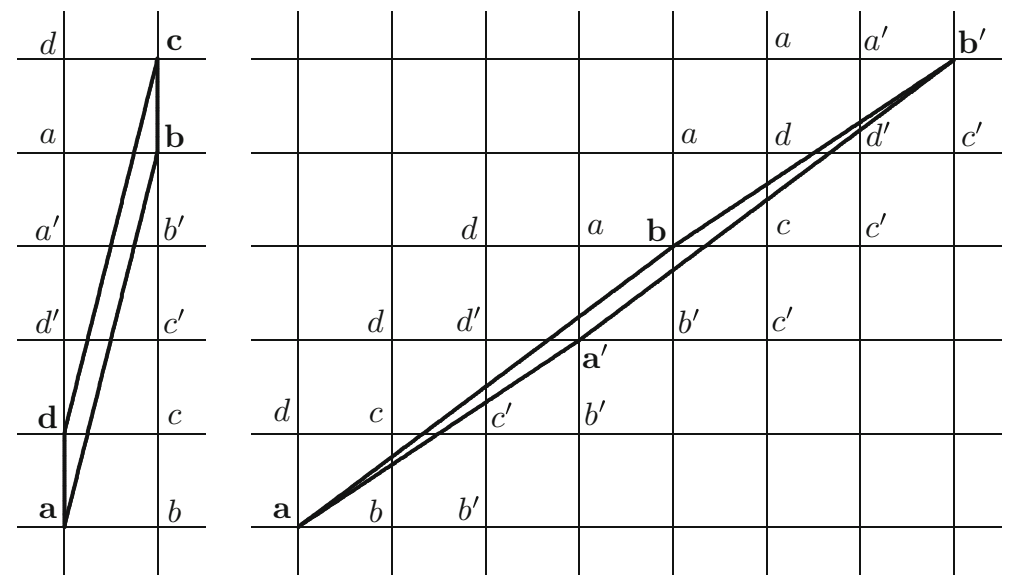

Fig. 6 To proof of Lemma 4.3

Lemma 4.3 (1) $V(C(p, q))=V(p, q)$. (2) The transformation $V(p, q) \mapsto$ $V(D(p, q))$ acts in the following way: $a \mapsto a, a^{\prime} \mapsto d \mapsto b \mapsto a^{\prime} b^{\prime} \mapsto d^{\prime} \mapsto c \mapsto$ $b^{\prime}, c^{\prime} \mapsto c^{\prime}$.

Proof Essentially, this theorem is proved in Fuchs (2014). The proof is contained in Fig. 6 [borrowed from Fuchs (2014)].

We can replace the standard fundamental square of the lattice by one of the parallelograms $(0,0),(1,4),(1,5),(0,1)$ or $(0,0),(3,2),(7,5),(4,3)$. The labeling of vertices shown in Fig. 6 provides labels for the vertices of these two parallelogram. In the left diagram, it is again $a b c d$, and this proves Part (1). In the right diagram, it is $a a^{\prime} b^{\prime} b$ which means that the whole labeling is transformed by the rotation of the cube which maps the face $a b c d$ into the face $a a^{\prime} b^{\prime} b$. This is the rotation of the cube by $120^{\circ}$ around the diagonal $c^{\prime} a$, as described in Part (2).

Now we formulate the main result of this section.

Theorem 4.4 Consider a simple geodesic segment on the cube emanating from the vertex a; suppose that it corresponds to some relatively prime $p, q$.

(1) (Davis et al. 2016, Corollary 5.15, Theorem 5.17) The vertex $V(p, q)$ cannot be the same as $a$; it is one of $a^{\prime}, b, d$ with the probability $\frac{4}{27}$ for each; one of $b^{\prime}, c, d^{\prime}$ with the probability $\frac{1}{9}$ for each and it is $c^{\prime}$ with the probability $\frac{2}{9}$.

(2) The vertex $V(p, q)$ is one of $a^{\prime}, b, d$, if $n_{C}(p, q)=4$, is one of $b^{\prime}, c, d^{\prime}$, if $n_{C}(p, q)=3$, and is $c^{\prime}$, if $n_{C}(p, q)=2$.

Proof As in the proof of Theorem 3.1, the main thing we need to prove is that a simple geodesic segment beginning at a vertex of the cube never ends at the same vertex. It is clear from the remark in the beginning of the section that if it the endpoint of the simple geodesic segment coincides with the beginning, then this segment corresponds to the pair $(p, q)$ with $p, q$ being both odd. 
Fig. 7 Vertices of the icosahedron

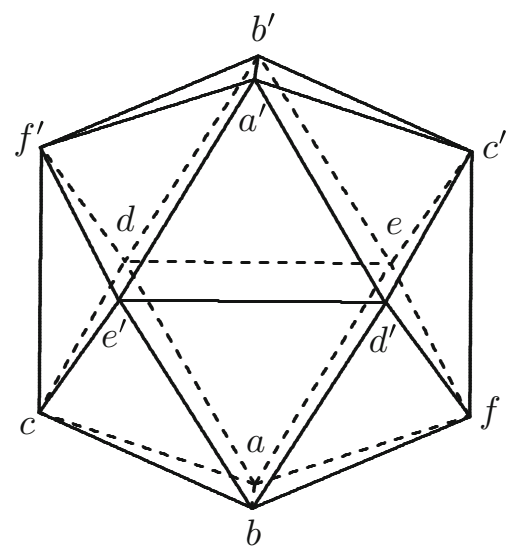

Return to Theorem 4.4. Notice that for arbitrary relatively prime odd $p, q$ there exists a sequence of transformations described before and in Lemma 4.3 which reduce the pair $(p, q)$ to $(1,1)$. Indeed, combining the transformation $C$ with sign changes and swapping of coordinates, we can reduce the general case to the case of positive $p, q$ with $p<q<2 p$ [besides the case $(p, q)=(1,1)$ ]; and this transformation does not increase the minimum of absolute values $|p|,|q|$. Then we apply $D ;(p, q)$ becomes $(4 p-3 q, 3 p-2 q)$, and $2 p<2 q<4 p \Rightarrow-p<3 p-2 q<q$, or $|3 p-2 q|<p$; thus the minimum of $|p|,|q|$ decreases. Repeating this procedure sufficiently many times, we arrive at $(p, q)=(1,1)$. Thus an arbitrary $(p, q)$ can be obtained from $(1,1)$ by a chain of inverse transformation, and, since $V(1,1)=c$ and no one of our transformations connects $a$ with anything else, we can conclude that $V(p, q) \neq a$.

Finally, consider an arbitrary simple geodesic segment $\sigma$ starting at $a$; let its planar development is $p \mathbf{u}+q \mathbf{v}$. Take the geodesic segment $\sigma^{\prime}$ parallel to $\sigma$ and starting at a point of the edge $a b$ close to $a$. If $\sigma$ ends at $b, d$, or $a^{\prime}$ then repeat $\sigma^{\prime} 4$ times; if $\sigma$ ends at $c, b^{\prime}$, or $d^{\prime}$, then repeat $\sigma^{\prime} 3$ times; and if $\sigma$ ends at $c^{\prime}$, then repeat $\sigma^{\prime} 2$ times. Obviously, we get a geodesic on the cube ending at a point of one of the edges $a b, a d$, or $a a^{\prime}$. But in the last two cases, to get a closed geodesic, we need to repeat already repeated 4 , or 3 , or 2 times $\sigma^{\prime} 3$ more times, which would mean that $n_{C}(p, q)=12$ or 9, or 6 , in contradiction to Part (3) of Theorem 4.1. Thus, the geodesic $\sigma^{\prime}$ repeated 4 , or 3 , or 2 times is closed and we conclude that if $V(p, q)$ is $b, d$, or $a^{\prime}$, then $n_{C}(p, q)=4$, if $V(p, q)$ is $c, b^{\prime}$ or $d^{\prime}$, then $n_{C}(p, q)=3$ and if $V(p, q)$ is $c^{\prime}$, then $n_{C}(p, q)=2$. Theorem 4.4 follows.

\section{Icosahedron}

We label the 12 vertices of the regular icosahedron as shown in Fig. 7. Notice that, here prime means "opposite": the vertex $a^{\prime}$ is opposite to $a$, the vertex $b^{\prime}$ is opposite to $b$, etc. 


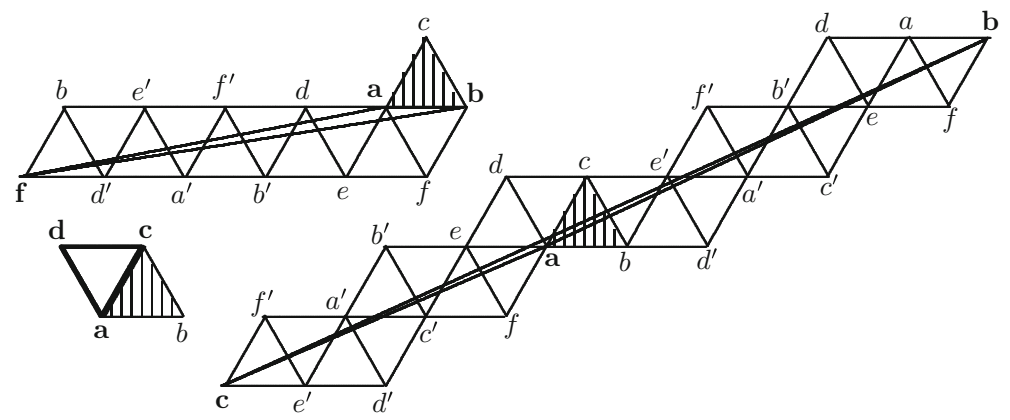

Fig. 8 To proof of Lemma 5.3

As in the cases of the tetrahedron and the octahedron, geodesics on the surface of the icosahedron (not passing through the vertices) are presented by straight lines in the plane furnished by the standard triangular tiling. A non-self-repeating closed geodesic corresponds to the segment $p \mathbf{u}+q \mathbf{v}$ with relatively prime integer $p, q$ repeated a certain number $n_{I}(p, q)$ of times.

Theorem 5.1 (Fuchs and Fuchs 2007, Theorem 6.1) The number $n_{I}(p, q)$ takes values 2, 3, and 5 .

The following informations of the function $n_{I}$ are contained in Fuchs (2014). Let $\mathcal{S}=\{(p, q) \in \mathbb{Z} \times \mathbb{Z} \mid \operatorname{GCD}(p, q)=1\} /(p, q) \sim(-p,-q)$. The group $P S L(2, \mathbb{Z})=S L(2, \mathbb{Z}) /\{ \pm I\}$ transitively acts in $\mathcal{S}$. Let $H$ be the subgroup of $P S L(2, \mathbb{Z})$ generated by $K=\left[\begin{array}{rr}0 & -1 \\ 1 & 1\end{array}\right], L=\left[\begin{array}{ll}-4 & 1 \\ -1 & 0\end{array}\right]$, and $M=\left[\begin{array}{ll}4 & -3 \\ 3 & -2\end{array}\right]$.

Theorem 5.2 (Fuchs 2014, Theorem 3.1)

(1) The group $H$ has index 10 in $\operatorname{PSL}(2, \mathbb{Z})$.

(2) The group $H$ has three orbits in $\mathcal{S}$, and these orbits are $\left\{n_{I}(p, q)=\right.$ $2\},\left\{n_{I}(p, q)=3\right\}$, and $\left\{n_{I}(p, q)=5\right\}$.

(3) The asymptotic sizes of these three orbits are related as 2:3:5.

As in the case of the cube, we need some enhancement of Theorem 5.2. For relatively prime $p, q$, let $V(p, q)$ be the vertex of the icosahedron, which is the endpoint of the simple geodesic segment beginning at $a$ and corresponding to the planar segment $p \mathbf{u}+q \mathbf{v}$.

Lemma 5.3 (1) $V(M(p, q))=V(p, q)$.

(2) The transformation $V(p, q) \mapsto V(K(p, q))$ is the rotation of the icosahedron $b \mapsto c \mapsto d \mapsto e \mapsto f \mapsto b, b^{\prime} \mapsto c^{\prime} \mapsto d^{\prime} \mapsto e^{\prime} \mapsto f^{\prime} \mapsto b^{\prime}$.

(3) The transformation $V(p, q) \mapsto V(L(p, q))$ is the rotation of the icosahedron inverse to the rotation in (2): $b \mapsto f \mapsto e \mapsto d \mapsto c \mapsto b, b^{\prime} \mapsto f^{\prime} \mapsto e^{\prime} \mapsto$ $d^{\prime} \mapsto c^{\prime} \mapsto b^{\prime}$. 
Proof As in the proof of Lemma 4.3, the transformations consist in replacements of the fundamental triangle of the tiling as shown in Fig. 8 [mostly borrowed from Fuchs (2014)]. We see that the transformations in Parts (1)-(3) of lemma correspond to the replacement of the fundamental triangle $a b c$ (shadowed in Fig. 8) by, respectively, triangles $a b c, a c d$, and $a f b$ (drawn in boldface lines in Fig. 8). Lemma follows.

The following statement is the main result of this section.

Theorem 5.4 Consider a simple geodesic segment on the icosahedron emanating from the vertex $a$ and ending at some vertex $h$; suppose that it corresponds to some relatively prime $p, q$.

(1) The vertex $h$ cannot be $a$.

(2) The vertex $h$ is one of the vertices $b, c, d, e, f$ with the probabilitiy $\frac{1}{10}$ for each; it is one of the vertices $b^{\prime}, c^{\prime}, d^{\prime}, e^{\prime}, f^{\prime}$ with the same probability $\frac{3}{50}$ for each; and it is $a^{\prime}$ with the probability $\frac{1}{5}$.

(3) The vertex $h$ is one of $b, c, d, e, f$, if $n_{I}(p, q)=5$, it is one of $b^{\prime}, c^{\prime}, d^{\prime}, e^{\prime}, f^{\prime}$, if $n_{I}(p, q)=3$, and it is $a^{\prime}$, if $n_{!}(p, q)=2$.

Proof Add to Lemma 5.3 that $V(-p,-q)$ is also obtained from $V(p, q)$ by a rotation, this time $b \mapsto e \mapsto c \mapsto f \mapsto d \mapsto b, b^{\prime} \mapsto e^{\prime} \mapsto c^{\prime} \mapsto f^{\prime} \mapsto d^{\prime} \mapsto b^{\prime}$. Consider the group $\widetilde{H} \subset S L(2 ; \mathbb{Z})$ generated by the matrices $-I, K, L, M$. The action of the group $\widetilde{H}$ in $\widetilde{\mathcal{S}}$ has three orbits, the inverse images the orbits of $H$ in $\mathcal{S}$ with resopect to the projection $\widetilde{\mathcal{S}} \rightarrow \mathcal{S}$. Lemma 5.3, supplemented by the last remark, shows that the sets $T_{A}=\{(p, q) \mid V(p, q) \in A$ with $A$ being one of the four sets $\{a\},\{b, c, d, e, f\},\left\{b^{\prime}, c^{\prime}, d^{\prime}, e^{\prime}, f^{\prime}\right\},\left\{a^{\prime}\right\}$ are invariant with respect to $\widetilde{H}$. Since the three orbits are represented by the pairs $(1,1),(1,2),(1,4)$ and $V(1,1)=$ $\left.c^{\prime}, V(1,2)=a^{\prime}, V 1,4\right)=d$, We conclude that $T_{\{a\}}$ is empty, while the three other sets $T_{A}$ are the three orbits of $\widetilde{H}$, and Theorem 5.2 yields identifications $T_{\{b, c, d, e, f\}}=$ $n_{I}^{-1}(5), T_{\left\{b^{\prime}, c^{\prime}, d^{\prime}, e^{\prime}, f^{\prime}\right\}}=n_{I}^{-1}(3), T_{\left\{a^{\prime}\right\}}=n_{!}^{-1}(2)$. Theorem follows.

\section{Dodecahedron}

I cannot say much about this case. Still it seems very likely that on the surface of the dodecahedron there exist simple geodesic segments which begin and end at the same vertex. By means of computer experiments, I have found a variety of likely examples of this. The shortest one is shown in Fig. 9. It is a 22-gonal geodesic emanating from the vertex $A$ under the angle $\alpha=\tan ^{-1} \frac{31 \sqrt{3-\phi}}{57 \phi-90} \approx 86.5^{\circ}$ with the side $(\phi=1.618 \ldots$ is the golden ratio). According to the computer, the distance between the 22nd edge and $A$ does not exceed $10^{-14}$ (we assume that the length of the side is 1 ). In the same 


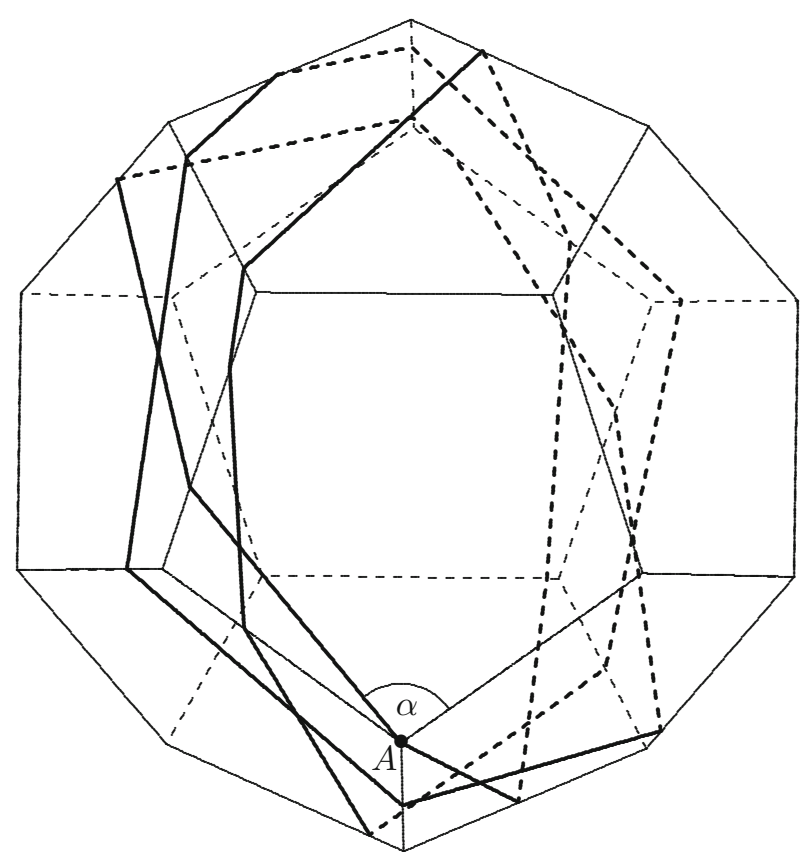

Fig. 9 A simple geodesic segment $A A$ on the dodecahedron

time, the distances between the other edges and other vertices are never less than 0.02 . This means that in the unlikely event that the 22nd edge does not hit $A$, we can make it ending at $A$ by a small perturbation of $\alpha$.

Acknowledgments This work was done during my stay at the Max Planck Institute in Bonn. I am grateful to the Institute for its hospitality.

\section{References}

Davis, D., Dods, V., Traub, C., Yang, J.: Geodesic trajectories on regular polyhedra. Discrete Math. (2016) (To appear)

Fuchs, D.: Periodic billiard trajectories in regular polygons and closed geodesics on regular polyhedra. Geom. Dedic. 170, 319-333 (2014)

Fuchs, D., Fuchs, E.: Closed geodesics on regular polyhedra. Mosc. Math. J. 7, 265-279 (2007) 